\title{
LA TRADIZIONE LETTERARIA SULLA PROSTITUZIONE SACRA A LOCRI EPIZEFIRI: I DUE VOTI DEL 477 E DEL 351-346 A.C.*
}

\author{
THE LITERARY TRADITION ABOUT THE SACRED PROSTITUTION IN LOCRI EPIZEFIRI:
} THE Two Vows of 477 AND 351-346 BC

IGNAZIO D'ANGELO

MEDITERRANEA UNIVERSITY OF REGGIO CALABRIA

L'esistenza della prostituzione sacra a Locri è stata oggetto di un intenso dibattito tra gli studiosi moderni: alcuni storici hanno negato che tale pratica fosse in vigore a Locri; ${ }^{1}$ altri, con argomenti diversi, ne hanno affermato la presenza. ${ }^{2}$

Il Van Compernolle ha sostenuto che la sacra prostituzione locrese è un'invenzione moderna alla cui base sta una maldestra combinazione e interpretazione delle fonti antiche (la II ode pitica di Pindaro e la tradizione letteraria relativa alla fine riservata dai Locresi ai familiari di Dionigi II): in particolare, il noto passo di Clearco (apud Athen. XII 515 E-516 A, fr. 43 Wehrli),

* A mia madre, Maria, che mi ha insegnato il valore della parola.

1 René VAN COMPERNOLLE, «Le tradizioni sulla fondazione e sulla storia arcaica di Locri Epizefirî e la propaganda politica alla fine del V secolo av. Cr. ", Annali della Scuola Normale Superiore di Pisa. Classe di Lettere e Filosofia, III ser., 6/2 (1976), p. 329-400, in part. p. 367-381; FRITZ GRAF, « Culti e credenze religiose della Magna Grecia », in Megale Hellas. Atti del XXI convegno di studi sulla Magna Grecia, Taranto, 2-5 ottobre 1981, Istituto per la storia e l'archeologia della Magna Grecia, Taranto 1982, p. 157-184, in part. p. 175-179; STEPHANIE Lynn Budin, The Myth of Sacred Prostitution in Antiquity, Cambridge University Press, Cambridge 2008, in part. p. 219.

2 Domenico MustI, «Problemi della storia di Locri Epizefirii », in Atti del XVI convegno di studi sulla Magna Grecia, Taranto 3-8 ottobre 1976, Arte Tipografica, Napoli 1977 (Atti del convegno di studi sulla Magna Grecia), p. 23-146, in part. p. 65-71; MARGHERITA GUARDUCCI, « Due pezzi insigni del museo nazionale romano: il 'Trono Ludovisi' e l"Acrolito Ludovisi' ", Bollettino d'arte, 33-34 (1985), p. 1-20, in part. p. 10; Domenico Musti, "Dall'età di Dionisio II fino all'occupazione romana (350-200 a. C.) » in SALVATORE SETTIS (ed.), Storia della Calabria antica, vol. II. Età italica e romana, Gangemi, Roma-Reggio Calabria 1994, p. 363-399, in part. p. 367; MARIO ToRELLI, «I culti di Locri ", in Atti del XVI convegno di studi sulla Magna Grecia, p. 147-184; LuIGI SANTI AmANTINI, "Ancora sulla prostituzione sacra a Locri Epizefirii », Miscellanea greca e romana, 9 (1984), p. 3962; Manuela Mari, "Tributo a Ilio e prostituzione sacra: storia e riflessi sociali di due riti femminili locresi », Rivista di cultura classica e medioevale, 2 (1997), p. 131-177.
\end{abstract}


che attesta la presenza della prostituzione nella Lidia, a Locri Epizefiri e a Cipro, si riferirebbe alla prostituzione profana, attestata a Locri e per la quale riporta a conferma un epigramma di Nosside (Anth. Palat. IX, 332). ${ }^{3}$

Anche il Graf ha ribadito l'inesistenza di tale pratica a Locri, ritenendo che i due testi letterari di Clearco (apud Athen. XII 515 E-516 A) e di Giustino (XXI 3), che hanno fatto pensare alla prostituzione sacra, «contengano solo la vaga memoria di una rituale deflorazione prenuziale $»^{4}$.

La Budin, che in generale ha negato con forza l'esistenza di ogni forma di prostituzione sacra nel mondo antico, nel caso specifico di Locri ritiene che, a parte Giustino, le altre fonti non menzionino alcuna pratica di prostituzione sacra: fra l'altro lo stesso racconto di Giustino apparirebbe poco coerente nella struttura narrativa. ${ }^{5}$

Il Musti, il Torelli, l'Amantini e la Mari, invece, hanno affermato non solo la storicità dei due voti pronunciati dai Locresi, quello del 477 all'epoca dell'attacco mosso da Anassila e dal figlio Leofrone contro Locri, e quello ripristinato per timore dei Lucani al tempo del soggiorno locrese di Dionigi II tra il 351 e il 346, ma anche la presenza abituale di una prostituzione sacra a Locri. ${ }^{6}$

Le differenti conclusioni, a cui sono giunti gli studiosi, impongono una rivisitazione e un'attenta analisi delle fonti letterarie al fine di chiarire i tanti dubbi in merito ad uno dei temi più controversi della storia di Locri Epizefiri.

La prima fonte in ordine cronologico che attesta la presenza di una forma di prostituzione a Locri è il peripatetico Clearco di Soli nell'isola di Cipro, che visse intorno alla metà del IV secolo a.C. e che scrisse opere di carattere erudito e filosofico.?

Il riferimento di Clearco alla prostituzione locrese $e^{8}$ è inserito all'interno di un

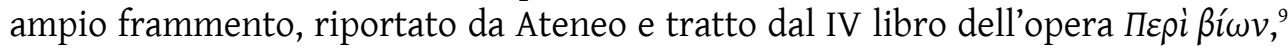

VAN COMPERNOLLE, « Le tradizioni », p. 367-381.

GRAF, «Culti e credenze religiose », p. 175-179.

Budin, « The Myth of Sacred Prostitution », p. 210 ss.

Musti, «Problemi della storia», p. 65-71; ToRelli, "I culti di Locri», p. 147-156; Amantini, "Ancora sulla prostituzione sacra », p. 39-62; MARI, "Tributo a Ilio », p. 131-177. Tra questi studiosi è comune la convinzione che i due voti di prostituzione sacra furono dettati da una occasione particolare perché è difficile credere che gli aristocratici prostituissero le loro donne abitualmente: è probabile - a loro avviso - che la prostituzione sacra fosse pertinente ad un livello più basso di classi.

7 La figura e l'opera di Clearco di Soli hanno attirato in questi ultimi anni l'interesse di tanti

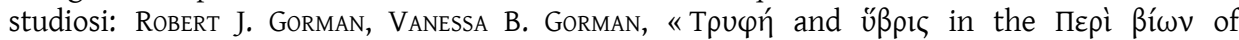
Clearchus », Philologus, 154 (2010), p. 187-208; STAVRos TsitsiRidis, Beiträge zu den Fragmenten des Klearchos von Soloi. Untersuchungen zur antiken Literatur und Geschichte, vol. CVII, De Gruyter, Berlin-Boston 2013 (Untersuchungen zur antiken Literatur und Geschichte, 107), p. 155-172; Gertjan Verhasselt, "What Were Works Пгрi Bíwv? A Study of the Extant Fragments", Philologus, 160 (2016), p. 59-83.

8 Clear. apud Athen. XII 516 A-B. 
in cui Clearco si sofferma sulla $\tau \rho v \varphi \eta ́$ dei Lidi e sull'origine della pratica della prostituzione in Lidia:

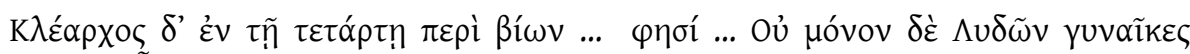

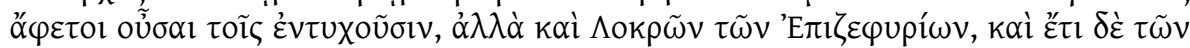

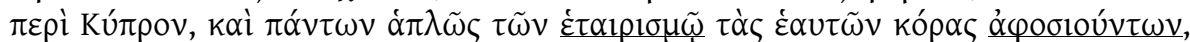

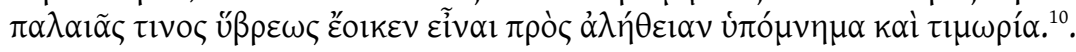

Nel IV libro delle Vite, Clearco [...] riferisce [...] non solo le donne dei Lidi erano lasciate libere ai primi capitati ma anche quelle dei Locresi Epizefiri e ancora quelle di Cipro e in generale di tutti quei popoli che consacrano le loro fanciulle alla prostituzione, e ciò sembra che sia in verità memoria e punizione di una qualche antica tracotanza [...].

Per il Torelli, la connessione di Locri con la Lidia e con Cipro, dove era attestata la prostituzione sacra legata al culto di Afrodite,${ }^{11}$ è un chiaro indizio della sua presenza anche a Locri: infatti questa forma di ierodulia era praticata a Koloe, a Tmolos e in città greche ${ }^{12}$ che avevano uno stretto contatto con la Lidia come per esempio Efeso, nell'isola di Cipro a Pafo e ad Amatunte ${ }^{13}$ e in Sicilia presso Erice. ${ }^{14}$

9 Clear. apud Athen. XII 515 E-516 B.

10 La tradizione manoscritta presenta $\tau \nmid \mu \omega \rho i ́ \alpha$ anche se alcuni studiosi hanno preferito emendare

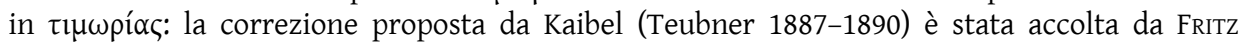
WeHrLI, Die Schule des Aristoteles, vol. III: Klearchos, Schwabe, Basel-Stuttgart 1969², p. 22 e da VAN COMPERNOLle, «Le tradizioni », p. 377-379. Non tutti gli storici hanno accolto l'emendamento: cfr. Musti, «Problemi della storia», p. 70-71, n. 25; ToRelli, «I culti di Locri», p. 150-151; AmantinI, "Ancora sulla prostituzione sacra », p. 39-40, n. 1; MARI, «Tributo a Ilio », p. 135, n. 17; VANia GHEZZI, «I tiranni siracusani e le vergini locresi », Parola del Passato, 338 (2004), p. 321-360, in part. p. 347, n. 117.

11 A Locri nell'area di Cento Camere, dove era venerata Afrodite, è stata rinvenuta una dedica arcaica a Cibele: la dea era oggetto di culto in Asia e, in particolare a Sardi, nella Lidia, era la dea del thalamos. In ambiente ionico come attesta Ipponatte (fr. 36 Diehl), Cibele era identificata con Afrodite. Cfr. TORELli, « I culti di Locri », p. 150; ID., « I culti », in SALVATORE SetTis (ed.), Storia della Calabria antica, vol. I, Gangemi, Roma-Reggio Calabria 1987, p. 589-612, in part. p. 599; AMANTINI, «Ancora sulla prostituzione sacra», p. 44. Sul culto di Afrodite a Locri cfr. MARCELLA BARRA BAgnasco, «Nuovi documenti sul culto di Afrodite a Locri Epizefiri », Parola del Passato, 250 (1990), p. 42-63.

12 Anche a Corinto era presente la prostituzione sacra come si può trarre conferma da una notizia di Pindaro (fr. 122 Snell) che ricorda il dono di ierodule da parte di Senofonte corinzio al santuario dell'Acrocorinto, dove si trovava anche un tempio di Afrodite. Sulla prostituzione a Corinto cfr. Gabriella AmiotTi, Corinto città libertina, Jesus, Edizioni San Paolo, Alba, 1984, p. 226231. Alcuni studiosi negano la presenza della prostituzione sacra nella città dell'Istmo. Cfr. GABRIELla PiRonti, "L'Afrodite di Corinto e il 'mito' della prostituzione sacra », in PAola Angeli BERNARDINI (ed.), Corinto. Luogo di azione e luogo di racconto. Atti del Convegno internazionale di Urbino, 23-25 Settembre 2009, Fabrizio Serra, Pisa-Roma 2013 (Quaderni urbinati di cultura classica. Atti di convegni, 9), p. 13-26.

13 TORELLI, « I culti di Locri », p. 150. 
Inoltre la forma verbale $\alpha \dot{\alpha} \varphi \sigma ı v u ́ v \tau \omega v$, che rimanda chiaramente ad un lessico sacrale, ci proietta verso quell'atmosfera religiosa nell'ambito della quale probabilmente doveva essere inserita tale pratica.

Nell'ottica di Clearco la prostituzione a Locri appare come un fatto abituale ${ }^{15}$

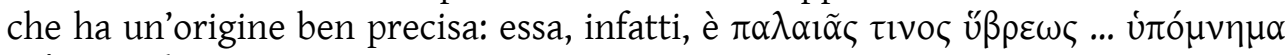

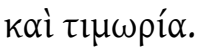

I moderni sono divisi riguardo al significato da dare all'espressione $\pi \alpha \lambda \alpha 1 \tilde{\alpha} \varsigma$

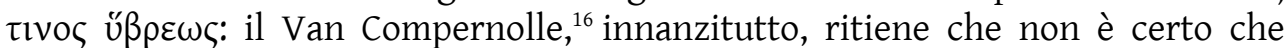

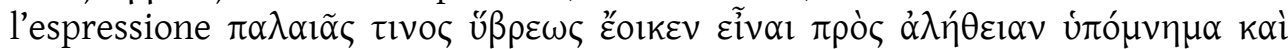
$\tau \iota \mu \omega p i ́ \alpha$ alluda ai Locresi e che comunque si tratta, pur sempre, di un'ipotesi

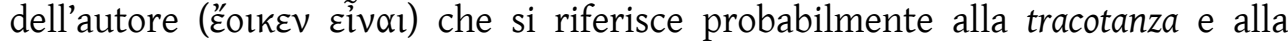
vendetta esercitata dai Locresi sulla famiglia di Dionigi II. ${ }^{17}$

Il Musti ${ }^{18}$, invece, ha interpretato la frase di Clearco nel senso che la prostituzione sacra sarebbe una specie di punizione inflitta alle discendenti dei servi locresi di Grecia, fondatori di Locri Epizefiri, ${ }^{19}$ che avevano portato con loro le donne nobili, con le quali si erano uniti durante l'assenza dei mariti e dei padri, accorsi in aiuto di Sparta, impegnata nella guerra contro i Messeni.

Allo stato attuale della ricerca storica non è possibile dire con sicurezza a che

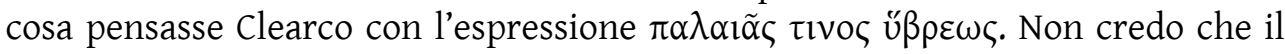
suo riferimento fosse diretto, come dice il Van Compernolle, alla strage della famiglia di Dionigi II per una ragione testuale e cronologica allo stesso tempo: $\mathrm{mi}$ domando, infatti, considerata la contemporaneità tra Clearco e il soggiorno locrese di Dionigi II, come nel pensiero di questo autore, quella üßpıৎ potesse apparire $\pi \alpha \lambda \alpha 1 \alpha$.

14 Beatrice Lietz, «La dea di Erice e il suo rapporto con la prostituzione », in Francesco GhizzANI Marcìa, Carolina Megale (eds.), Materiali per Populonia, 8, ETS, Pisa 2009, p. 247-254.

15 Cfr. AmANTini, « Ancora sulla prostituzione sacra », p. 40.

16 VAN COMPERNOLLE, « Le tradizioni », p. 378-379.

17 Da notare che l'interpretazione del Van Compernolle è determinata dalla preferenza della

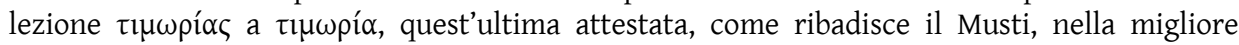
tradizione manoscritta (cod. A e B). Cfr. Musti, « Problemi della storia », p. 70, n. 25.

18 Musti, «Problemi della storia », p. 37 ss. e p. 70, n. 25.

19 Sulla fondazione di Locri Epizefiri ci sono giunte due tradizioni: una aristotelica, che affermava l'origine servile dei coloni, l'altra timaica, secondo la quale i fondatori avevano un'origine libera. Non è questa la sede per affrontare questa problematica per cui rimando ai seguenti contributi: VAN COMPERnolle, « Le tradizioni », p. 330-353; Musti, « Problemi della storia », p. 3765; LoRedana Cappelletti, «Colonizzazioni al femminile: il caso di Locri Epizefiri », in Mariavaleria Del Tufo, Francesco lucrezi (eds.), lo spazio della donna nel mondo antico, Atti del Seminario di Studi, Napoli, Università Suor Orsola Benincasa, Centro Studi sui Fondamenti del Diritto antico, 22 maggio 2017, Editoriale scientifica, Napoli 2019 (Fondamenti del diritto antico, 6), p. 213-232. 
Interessante per un chiarimento del problema è un testo di Licofrone (Alex., 1141-73), ${ }^{20}$ dove Cassandra profetizza sofferenze per le giovani donne locresi (sta parlando dei Locresi di Grecia), che verranno inviate ad Ilio al tempio di Atena per mille anni come serve a riparazione della colpa di Aiace, ${ }^{21}$ che durante il sacco di Troia aveva fatto violenza su di lei (1155-1159):

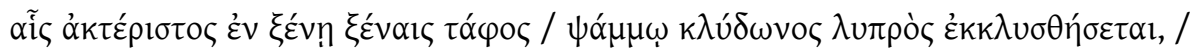

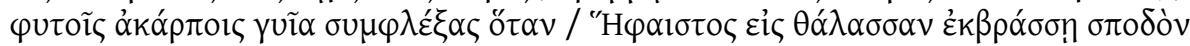

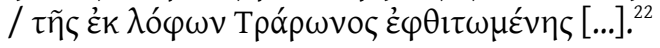

Per loro, straniere in terra straniera, una tomba dolente, / priva di esequie, sarà lavata via dall'onda sabbiosa, / quando bruciando le membra con piante infeconde / Efesto getterà in mare le ceneri / di colei che perì dalle cime del Trarone [...]. ${ }^{23}$

A mio avviso, il testo di Licofrone è in stretto rapporto con l'affermazione di Clearco secondo cui la prostituzione, propria dei Locresi Epizefiri (al pari dei Lidi e dei Ciprioti), sia in verità memoria e punizione di una qualche antica tracotanza

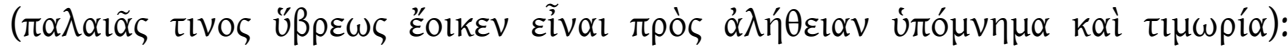
l'antica colpa, dunque, potrebbe essere per i Locresi quella di Aiace Oileo che aveva violentato Cassandra all'epoca della conquista di Troia.

Nel leggere il testo di Licofrone sono stato colpito dal riferimento all'incinerazione e alla dispersione in mare delle giovani locresi (ma dei Locresi di Grecia) che ricorda il trattamento a cui furono sottoposti i familiari di Dionigi II, dopo che essi furono violentati e uccisi secondo quanto ci viene riferito dallo

20 Cfr. Dennis D. Hughes, I sacrifici umani nell'antica Grecia, Salerno, Roma 1999 (Piccoli saggi, 5), p. 265 ss.

21 La figura di Aiace Oileo è strettamente collegata ai Locresi Epizefiri a proposito della battaglia della Sagra dal momento che, secondo la leggenda, Aiace sarebbe intervenuto a fianco dei Locresi nello scontro contro i Crotoniati. Cfr. René VAn Compernolle, « Ajax et les Dioscures au secours des Locriens sur les rives de la Sagra (ca. 575-565 av. notre ère) », in Hommages à Marcel Renard, vol. II, Latomus, Bruxelles 1969, p. 733-766. MARTA SORDI, « La leggenda dei Dioscuri nella battaglia della Sagra e di Lago Regillo », Contributi dell'Istituto di storia antica, 1 (1972), p. 47-70.

22 Questi versi sono presenti anche in Tzetze (Chil. 5 728-745) dove l'erudito bizantino del XII secolo ricorda che nella Ionia, quando una sventura colpiva una città per la collera divina, veniva preso l'uomo più ripugnante e, dopo aver preparato il luogo del sacrificio e avergli dato in mano formaggio, focaccia e fichi secchi e dopo averlo frustato, veniva bruciato su una pira e le sue ceneri venivano disperse in mare come anche - dice Tzetze - Licofrone riferisce in qualche luogo delle giovinette locresi... (segue con qualche variante irrilevante il passo di Licofrone sopra riportato).

23 La traduzione del passo di Licofrone è stata riportata dall'opera di HugHES, I sacrifici umani, p. 265. 
Ignazio D'Angelo

stesso Clearco (apud Athen. XII 541 C-E fr. 47 Wehrli), da Eliano (Var. Hist. IX, 8) e da Strabone (VI 18 C 259). ${ }^{24}$

Sia Clearco sia Eliano, dopo aver ricordato l'arrivo di Dionigi II a Locri, si soffermano su una strana scena in cui il tiranno è impegnato a rotolarsi nudo con donne nude su un tappeto di fiori nella casa più grande della città: per questa sua colpa i Locresi costrinsero la moglie e le figlie di Dionigi II a prostituirsi, le violentarono e, inserendo aghi dentro le unghie delle mani, le uccisero; mangiarono una parte dei loro corpi e il resto venne buttato in mare.

Nel suo racconto Strabone aggiunge altri particolari relativi al soggiorno locrese del tiranno: ricorda lo ius primae noctis esercitato nei confronti delle donne locresi, presenta un'altra curiosa scena in cui delle fanciulle nude, con sandali non legati, uno più alto, l'altro più basso, sono costrette da Dionigi a inseguire delle colombe, e infine si sofferma sulla violenza esercitata dai Locresi sui familiari del tiranno, che vengono violentati, uccisi, bruciati e le loro ceneri disperse in mare. ${ }^{25}$

L'ipotesi che Clearco, Eliano e Strabone abbiano arricchito i loro resoconti sull'uccisione della moglie e dei figli del tiranno con richiami a quei racconti che coinvolgevano le fanciulle dei Locresi di Grecia, ${ }^{26}$ costrette a recarsi a Troia a servire nel tempio di Atena, prende sempre più consistenza se collegata alla prostituzione sacra, a cui questi testi sembrano in qualche modo alludere: l'episodio riferito da Clearco ed Eliano relativo ai giochi erotici sul tappeto di fiori nella casa più grande di Locri, la scena descritta da Strabone in cui le fanciulle nude inseguono delle colombe hanno portato il Prückner a vedere i residui della tradizione di una festa di Afrodite a Locri. ${ }^{27}$

Anche il Torelli, collegando il gioco delle colombe alla descrizione del pavimento cosparso di fiori nella casa più grande di Locri (Athen. XII 541 C-D-E; Ael. Var. Hist. IX, 8), ritiene che l'uno e l'altro si inseriscano nel clima della religiosità locrese: ${ }^{28}$ la menzione da parte di Giustino del die festo Veneris conferma, a mio avviso, questa ipotesi e credo che questi momenti facciano parte proprio del cerimoniale del voto di prostituzione sacra, di cui parla Giustino.

24 Per uno sguardo sinottico di questi racconti e per una loro discussione cfr. IGNAZIo D’ANGELO, « La tradizione letteraria sul soggiorno locrese di Dionigi II: un frammento sconosciuto di Timeo (Strab. VI 1, 8, C 259) », Rivista dell'Istituto Lombardo, 136/1 (2002), p. 177-191.

25 Nel testo di Strabone è assente il particolare del cannibalismo, presente nella tradizione letteraria confluita nel racconto di Clearco ed Eliano.

26 Sulla vicenda delle fanciulle locresi e il rapporto con i sacrifici umani cfr. Hughes, I sacrifici umani, p. 263-289; PIERRE BONNECHERE, Le sacrifice humain en Grèce ancienne, Centre international d'étude de la religion grecque antique, Athènes-Liège 1994 (Kernos. Supplément, 3), p. 150-163.

27 Cfr. HeLmUT PRÜCKNER, Die lokrischen Tonreliefs. Beitrag zur Kultgeschichte von Lokroi Epizephyrioi, Philipp von Zabern, Mainz am Rhein 1968, p. 8-14.

28 TORELLI, « I culti di Locri », p. 182. 
Un altro testo che sembra inserirsi nell'atmosfera religiosa, di cui stiamo parlando, è il noto epigramma della poetessa locrese Nosside (Anth. Pal. IX, 332): ${ }^{29}$

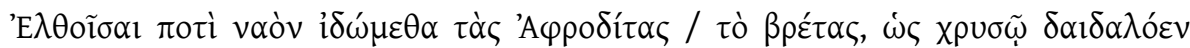

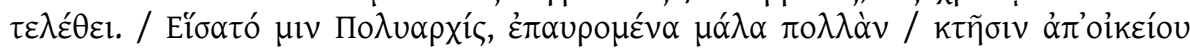

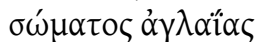

Giunte nel tempio guardiamo la statua di Afrodite / che appare scolpita in oro. / La innalzò Poliarchide che guadagnò molte ricchezze / dalla bellezza del proprio corpo. ${ }^{30}$

29 Originaria di Locri visse intorno al 300 a. C. Su Nosside cfr. Marcello GIGANTE, « Nosside », Parola del Passato, 154-155 (1974), p. 22-39. In questo articolo l'autore commenta i dodici epigrammi di Nosside sulla base di un confronto con Saffo, Ibico di Reggio, Leonida di Taranto e altri poeti greci.

30 Alcuni studiosi hanno pensato di collegare l'epigramma di Nosside con le tavolette 23, 30 e 31 dell'archivio del tempio di Zeus a Locri, nelle quali compare uno iapã $\nu \mu$ í $\sigma \omega \omega \mu \alpha$, che è stato considerato dal De Franciscis e dal Torelli come voce riferibile alla prostituzione sacra: in particolare il De Franciscis ha inteso l'espressione i $\alpha \rho \tilde{\alpha} v \mu$ í $\sigma \theta \omega \mu \alpha$ come «mercede delle sacerdotesse »; il Torelli ha fatto notare la corrispondenza tra la cifra di venti talenti della voce i $\alpha \rho \tilde{\alpha} v \mu$ í́$\theta \omega \mu \alpha$ delle tavolette e il numero degli oikoi della Stoá ad U di Centocamere, identificata dal Torelli con il lupanare del testo di Giustino (XXI 3), dove Dionigi II aveva proposto ai Locresi di far assolvere il voto di prostituzione sacra; l'Amantini ha richiamato un passo di Ateneo (XIII 581 A) dove il termine $\mu$ í $\sigma \omega \mu \alpha$ significa proprio "prezzo di una prostituta ». Significativa è l'osservazione del Gigante (Nosside, cit., in part. 31-32) secondo cui la $\kappa \tau \tilde{\eta} \sigma ı v$ dell'epigramma di Nosside, sopra riportato, richiami il $\mu$ í $\sigma \omega \mu \alpha$ delle tavole locresi. Tuttavia non tutti gli storici accettano lo i $\alpha \rho \tilde{\alpha} v \mu$ í $\sigma \theta \omega \mu \alpha$ delle tavolette come voce riferibile alla prostituzione sacra: lo stesso Gigante, che in un primo momento aveva accettato l'interpretazione data dal De Franciscis, successivamente se ne è allontanato, proponendo di intendere lo i $\alpha \rho \tilde{\alpha} \nu \mu i ́ \sigma \theta \omega \mu \alpha$ come un riferimento a terre sacre; il Costabile, infatti, ha interpretato lo i $\alpha \rho \tilde{\alpha} v \mu$ í $\sigma \theta \omega \mu \alpha$ come canone di affitto di terre sacre. È dunque difficile utilizzare le tavolette come prova per l'esistenza della prostituzione sacra a Locri, considerata l'ambiguità dell'espressione i $\alpha \rho \tilde{\alpha} v \mu$ í $\sigma \omega \omega \mu \alpha$. Cfr. Alfonso De Franciscis, Stato e società in Locri Epizefiri: l'archivio dell'olympieion locrese, Libreria scientifica, Napoli 1972 (Pubblicazioni a cura del Centro studi della Magna Grecia della Università di Napoli, n. s., 3), p. 151-158; ID., « Le tavole di Locri negli studi dal 1972 ad oggi », in Domenico Musti (ed.), Le Tavole di Locri. Atti del colloquio sugli aspetti politici, economici, cultuali e linguistici dei testi dell'archivio locrese (Napoli 26-27 aprile 1977), Edizioni dell'Ateneo \& Bizzarri, Roma 1979, p. 15-36, in part. p. 31-33; MARIO TORELLI, «Considerazioni sugli aspetti religiosi e cultuali », in Domenico Musti (ed.), Le Tavole di Locri, p. 91-112, in part. p. 104-106; AMANTINI, « Ancora sulla prostituzione sacra », p. 55-62; GIGANTE, « Nosside », p. 3132; ID., « Le tavole di Locri come testo storico », in Domenico Musti (ed.), Le Tavole di Locri, p. 3759, in part. p. 43-45; discussione p. 114-115; Felice COSTABILE, « Redditi, terre e fonti finanziarie dell'Olympieion: tributi, imposte e rapporti contrattuali », in FeLICE COSTABILE (ed.), Polis ed Olympieion a Locri Epizefiri. Costituzione economia e finanze di una città della Magna Grecia. Editio altera e traduzione delle tabelle locrese, Rubbettino, Soveria Mannelli (CZ) 1992, p. 160-174, in part. p. 162; Felice Costabile, "Santuari e culti a Locri Epizefiri», in Enigmi delle civiltà antiche dal Mediterraneo al Nilo, vol. I, Iiriti, Reggio Calabria 2007, p. 97-105, in part. p. 102. 
L'epigramma di Nosside non lascia adito a dubbi: la statua in oro di Afrodite si presenta come un ex voto offerto da questa donna di nome Poliarchide, che evidentemente ha guadagnato ricchi compensi grazie alla bellezza del suo corpo e, dunque, all'attività di etera svolta all'interno del tempio.

Il fatto che tale statua si trovi in un tempio e che l'epigramma di Nosside sia inserito in un'atmosfera religiosa nell'ambito del culto ad Afrodite, credo che concorra a confermare la presenza della prostituzione sacra a Locri legata ad Afrodite, come d'altra parte viene confermato dal racconto di Giustino (XXI, 3) che inserisce il voto del 477 in rapporto ad una festa in onore di Venere (Cum Reginorum tyranni Leophronis bello Locrenses premerentur, voverant, si victores forent, ut die festo Veneris virgines suas prostituerent).

Proprio il testo di Giustino (XXI, 3), relativo al soggiorno locrese del tiranno Dionigi II, contiene espliciti riferimenti alla prostituzione sacra: in esso, infatti sono ricordati sia il voto risalente all'attacco reggino contro Locri nel 477 a.C. sia quello che cronologicamente si colloca durante il periodo di permanenza del tiranno siracusano a Locri (351-346 a.C.).

Alcuni studiosi moderni, confrontando il racconto di Giustino con altre fonti, sono giunti alle seguenti conclusioni soprattutto per quanto riguarda la natura di questi due voti.

Per l'Amantini, ${ }^{31}$ la Mari ${ }^{32}$ e il Cordiano, ${ }^{33} \mathrm{i}$ due voti di prostituzione sacra ebbero un carattere di eccezionalità ${ }^{34}$ per due ragioni: innanzitutto per le circostanze di pericolo in cui furono pronunciati (l'attacco reggino del 477 a.C. contro Locri e una sconfitta locrese ad opera dei Lucani intorno alla metà del IV sec. a.C.) e poi per il coinvolgimento di donne di rango elevato. In particolare il voto del 477 non sarebbe stato adempiuto (come suggerirebbe l'espressione di Giustino 'quo voto intermisso') ${ }^{35}$, grazie all'intervento diplomatico del tiranno siracusano Ierone, che, liberando le fanciulle locresi da un così pesante tributo, ne ottenne così quella gratitudine, a cui sembra alludere Pindaro nella II Pitica. ${ }^{36}$

31 AmANTINI, « Ancora sulla prostituzione sacra », p. 49 ss.

32 MARI, « Tributo a Ilio », p. 146 ss.

33 Cfr. GiusepPe CoRdiano, «La fine della 'ierodulia' femminile a Temesa magno greca nella propaganda dei Locresi Epizefirii», ARYS. Antigüedad: Religiones y Sociedades, 3 (2000), p. 115-127, in part. p. 118 ss.

34 La straordinarietà del voto si potrebbe comprendere ammettendo la presenza abituale a Locri di forme di prostituzione sacra che non coinvolgevano donne aristocratiche, ma elementi appartenenti a livelli più bassi della popolazione. Cfr. MusTI, «Problemi della storia », p. 71; AMANTINI, « Ancora sulla prostituzione sacra », p. 47.

35 Iust. XXI 3. Sulla mancata attuazione del voto del 477 a.C. si sono espressi diversi studiosi: VAN COMPERNolle, «Le tradizioni », p. 371-372; MustI, « Problemi della storia », p. 66; MARI, « Tributo a Ilio », p. 143, 146; GHEZzI, « I tiranni siracusani », p. 339.

36 Cfr. AmANTINI, «Ancora sulla prostituzione sacra », p. 42 ss.; GuARDuccI, « Due pezzi insigni», p. 12; MARI, "Tributo a Ilio », p. 151; CoRDIANo, «La fine della 'ierodulia' femminile », p. 119. Anche il ToRell («I culti », p. 599) pensa di trovare un'eco del votum nell'ode di Pindaro. Al 
Il voto di metà IV secolo avrebbe avuto, invece, un carattere simbolico, ${ }^{37}$ stante al racconto dello stesso Giustino, che precisa che nessuno degli uomini avrebbe dovuto violare le donne convenute nel lupanare: il ripristino del voto ad opera di Dionigi II, giustificato da una sconfitta subita dai Locresi ad opera dei Lucani, in realtà fu un espediente con il quale Dionigi si appropriò delle ricchezze delle donne aristocratiche.

Le interessanti osservazioni contenute in questi studi e i risultati di alcune mie ricerche condotte negli anni passati sull'attacco reggino del 477 a.C. e sul soggiorno locrese di Dionigi II ${ }^{38}$ costituiscono dei punti di partenza imprescindibili per tentare di aprire uno spiraglio sulla complessa struttura narrativa del racconto trogiano, di cui ritengo opportuno riportare il testo e la traduzione.

Giustino (XXI, 2, 8; 3) inserisce la ripresa del voto da parte di Dionigi in quei provvedimenti finalizzati a rimpinguare le finanze del tiranno: ${ }^{39}$

Qui cum obsidionem arcis timeret, cum omni regio apparatu in Italiam profugit tacitus. Exul Locrensibus sociis acceptus, velut iure regnaret, arcem occupat solitamque sibi saevitiam exercet. Coniuges principum ad stuprum rapi iubebat, virgines ante nuptias abducebat stupratasque procis reddebat, locupletissimos quosque aut civitate pellebat aut occidi imperabat bonaque eorum invadebat. Dein cum rapinae occasio deesset, universam civitatem callido commento circumvenit. Cum Reginorum tyranni Leophronis bello Locrenses premerentur, voverant, si victores forent, ut die festo Veneris virgines suas prostituerent. Quo voto intermisso cum adversa bella cum Lucanis gererent, in contionem eos Dionysius vocat; hortatur, ut uxores filiasque suas in templum Veneris quam possint ornatissimas mittant, ex quibus sorte ductae centum voto publico fungantur religionisque gratia uno stent in lupanari mense omnibus ante iuratis viris, ne quis ullam adtaminet. Quae res ne virginibus voto civitatem solventibus fraudi esset, decretum facerent: ne qua virgo nuberet, priusquam illae maritis traderentur.

contrario la SOURVINOU-INWOOD ( $"$ The votum of 477/6 B. C. and the foundation legend of Locri Epizephyrii », The Classical Quarterly, 24 (1974), p. 186-198, in part. p. 187, n. 4) aveva escluso che Pindaro conoscesse il votum. Così anche MARCELlo GIGANTE, "Pindaro quale testimone della civiltà letteraria e agonale di Locri Epizefiri », Klearchos, 20 (1978), p. 59-73, in part. p. 67.

37 Van Compernolle, «Le tradizioni », p. 373; Musti, «Problemi della storia», p. 67; Amantini, "Ancora sulla prostituzione sacra », p. 49.

38 Cfr. IGNAZIo D'ANGELo, «L'attacco reggino del 477 a.C. contro Locri e l'intervento di Ierone», Aevum, 76/1 (2002), p. 9-15; D’ANGELo, « La tradizione letteraria », p. 177-191.

39 Cfr. Federicomaria Muccioli, Dionisio II. Storia e tradizione letteraria, CLUEB, Bologna 1999 (Monografie di Simblos, 1), p. 352-353; JoACHIM LosehAND, "Votum (publicum) intermissum: Bemerkungen zur 'sakralen Prostitution' in Lokroi Epizephyrioi », in TANJA S. SCHEER, MARTIN LINDNER (eds.), Tempelprostitution im Altertum. Fakten und Fiktionen, Verlag Antike, Berlin 2009 (Oikumene. Studien zur antiken Weltgeschichte, 6) p.267-292, in part. p. 271; LOREDANA Cappelletti, «Esclusive notizie locresi in Nosside (Anth. Pal. 6.132 E 265) », Athenaeum, 106/2 (2018), p. 474-490. 
Probato consilio, quo et superstitioni et pudicitiae virginum consulebatur, certatim omnes feminae inpensius exornatae in templum Veneris conveniunt, quas omnes Dionysius inmissis militibus spoliat ornamentaque matronarum in praedam suam vertit. Quarundam viros ditiores interficit, quasdam ad prodendas virorum pecunias torquet. Cum his artibus per annos sex regnasset, conspiratione Locrorum civitate pulsus in Siciliam redit [...].

Dionigi, temendo l'assedio della rocca, fuggì di nascosto in Italia con tutto l'apparato regale. Esule, accolto dai Locresi suoi alleati, come se regnasse di diritto, occupò l'acropoli ed esercitò l'abituale crudeltà. Comandava di far stuprare le mogli dei più illustri, sottraeva le fanciulle prima delle nozze e le restituiva disonorate ai mariti, cacciava i cittadini più ricchi dalla città o ordinava di ucciderli e si appropriava delle loro ricchezze. Quindi, non trascurando occasione di rapina, raggirò tutta la cittadinanza con un ingegnoso disegno. I Locresi, essendo assediati dalla guerra di Leofrone, tiranno di Reggio, avevano fatto voto di prostituire le loro fanciulle nel giorno di festa di Venere, se fossero risultati vincitori. Ma avendo interrotto questo voto e poiché combattevano guerre sfavorevoli con i Lucani, Dionisio chiama i cittadini locresi in assemblea; li esorta a mandare nel tempio di Venere le proprie figlie e le proprie mogli, ${ }^{40}$ ornate il più possibile; ed estrattene a sorte cento ${ }^{41}$ le esorta ad assolvere il voto pubblico e a rimanere un mese nel lupanare per dovere religioso, avendo fatto giurare a tutti gli uomini che nessuno di loro ne avrebbe violata una. Affinché ciò non recasse danno alle fanciulle che liberavano la città dal voto, li esortò ad approvare un decreto, secondo cui nessuna fanciulla si sarebbe sposata prima che quelle avessero trovato marito. Approvata la proposta, secondo cui si deliberava a favore della religione e dell'onore delle fanciulle, a gara tutte le donne, ornate con molta cura, convennero al tempio di Venere, dove Dionigi, avendo mandato i mercenari, le spogliò tutte e fece bottino dei gioielli delle matrone. Fece uccidere gli uomini più ricchi di alcune donne, ne torturò altre affinché rivelassero le ricchezze dei loro uomini. Poiché regnò con questi sistemi per sei anni, ${ }^{42}$ espulso da una congiura cittadina, ritornò in Sicilia.

Sebbene in generale l'opera storica di Trogo-Giustino ponga problemi interpretativi di notevole entità soprattutto in merito all'identità dei due autori,

40 La teoria del Graf secondo cui dietro il racconto di Giustino ci troveremmo di fronte ad un rituale di deflorazione è in realtà in contraddizione con lo stesso racconto, dove la proposta di Dionigi all'assemblea locrese non riguarda soltanto fanciulle, ma anche donne già sposate (uxores filiasque). Cfr. GRAF, « Culti e credenze religiose », p. 175-179.

41 Il numero di donne estratte sembra suggerire un rapporto tra la nobiltà locrese e le donne, appartenenti alla 'cento casate' della madrepatria Locride, che parteciparono alla fondazione di Locri Epizefiri. Cfr. ToRelli, « I culti di Locri », p. 152; CAPPELlETTI, « Esclusive notizie », p. 488.

42 Sulla durata del soggiorno locrese di Dionigi II cfr. PIERO MELONI, « Il soggiorno di Dionisio II a Locri », Studi italiani di filologia classica, 25 (1951), p. 149-168; Musti, « Problemi della storia », p. 99; IGNAZIo D'ANGELo, « Le tavolette di Locri e il regnum di Dionigi II », Aevum, 75/1 (2001), p. 9-24, in part. p. 19, n. 58; GIUSEPPE ZecchinI, in Justin, Abrégé des Histoires Philippiques de Trogue Pompée, t. II: Livres XI-XXIII, Les Belles Lettres, Paris, 2018, p. 232. 
alla natura dell'epitome, al rapporto tra Giustino e Trogo e al problema delle fonti ${ }^{43}$ e sebbene in questo passo siano state rilevate opportunamente una serie di incongruenze narrative e delle contraddizioni, ${ }^{44}$ tuttavia non si può non tenere conto delle informazioni sui due voti contenute in Giustino soprattutto sulla base di un confronto-riscontro con la tradizione letteraria confluita in Clearco-Eliano ${ }^{45}$ e in quella di Strabone. ${ }^{46}$

Secondo il racconto di Giustino, al tempo dell'attacco di Leofrone, tiranno di Reggio, i Locresi avevano pronunciato il voto di prostituire le loro fanciulle in occasione di una festa in onore di Venere, se avessero vinto il conflitto.

La struttura del cum narrativum stabilisce un nesso temporale-causale tra l'attacco reggino e il pronunciamento del voto e pertanto risulta importante riflettere sull'esito di questa guerra, che ebbe delle ripercussioni sulle relazioni tra la tirannide dinomenidea e le città italiote. ${ }^{47}$

43 Per un quadro generale sull'epitome di Giustino cfr. Cinzia Bearzot, Franca Landucci (eds.), Studi sull'Epitome di Giustino I. Dagli Assiri a Filippo II di Macedonia, Vita e Pensiero, Milano 2014 (Contributi di storia antica, 12); EAD., Studi sull'Epitome di Giustino II. Da Alessandro Magno a Filippo $V$ di Macedonia, Vita e Pensiero, Milano 2015 (Contributi di storia antica, 13); AlEssandro Galimberti, Giuseppe Zecchini (ed.), Studi sull'Epitome di Giustino III. Il tardo ellenismo. I Parti e i Romani, Vita e Pensiero, Milano 2016 (Contributi di storia antica, 14); DAGMAR Hofman, Griechische Weltgeschichte auf Latein. Iustins 'Epitoma historiarum Pompei Trogi' und die Geschichtskonzeption des Pompeius Trogus, F. Steiner, Stuttgart 2018 (Hermes Einzelschriften, 114); ALICE BorGNA, Ripensare la storia universale. Giustino e l'Epitome delle 'Storie Filippiche' di Pompeo Trogo, Olms, HildesheimZürich-New York 2018 (Spudasmata, 176).

44 Cfr. GHEZzI, «I tiranni siracusani », p. 330-331.

45 Clear. apud Athen. XII 541 C-E fr. 47 Wehrli; Ael. Var. Hist. IX, 8.

46 Strab. VI 18 C 259.

47 L'intervento del tiranno Ierone a favore di Locri faceva parte di un progetto politico-territoriale più ampio finalizzato ad ampliare la sfera d'influenza dei Dinomenidi nell'area italiota come rivelano alcuni provvedimenti attuati tra il 480 e il 470 a.C.: la costruzione di un edificio sacro chiamato corno di Amaltea nei pressi di Ipponion (colonia locrese) ad opera di Gelone (Athen. XII 542, a=FgrHist II A, 144), l'intervento militare di Ierone a fianco dei Sibariti, attaccati da Crotone (Diod. XI 48, 3-5; Schol. Pind. Ol. II 29 bcd); il sostegno siracusano dato ai Locresi nella conquista di Temesa (Strab. VI 1, 5 C 255) e della fascia ionica tra l'Halex e il Kaikinos; la vittoria siracusana sugli Etruschi a Cuma; l'allontanamento del reggino Micito, che aveva tentato di rilanciare la politica imperialistica di Reggio. Cfr. GIovanna De Sensi SeSTITO, « I Dinomenidi, nel basso e medio Tirreno fra Imera e Cuma ", in Mélanges de l'École française de Rome. Antiquité, 93 (1981-1982), p. 617-642; Nino LuRAGHI, Tirannidi arcaiche in Sicilia e Magna Grecia. Da Panezio di Leontini alla caduta dei Dinomenidi, Olschki, Firenze 1994 (Fondazione Luigi Firpo. Centro di studi sul pensiero politico. Studi e testi, 3), p. 273-373; Giovanni Millino, « Micito di Reggio e la symmachia con Taranto ", Hespería, 17 (2003), p. 207-221; IgnAzio D’Angelo, « La politica espansionistica di Locri Epizefirii negli anni compresi fra il 477 e il 467 a.C.: la versione locrese della conquista di Temesa (Strab. VI 1, 5 C 255)», Polis. Studi interdisciplinari sul mondo antico, 3 (2010), p. 27-34; GIovanNa De Sensi SeSTITo, « Temesa: tradizioni e documentazioni a confronto », in LuCIA FERNANDA RUFFo (ed.), Verso Temesa: Storia e prospettive di una ricerca. Atti del convegno, Campora San Giovanni-Amantea, 31 ottobre 2015, Mariano Spina, San Giovanni in Fiore (CS) 2016, p. 9-32. 
Ignazio D'Angelo

È communis opinio tra gli studiosi moderni che non vi sia stato alcun attacco reggino ${ }^{48}$ ma solo una minaccia di guerra da parte di Reggio, risolta grazie all'intervento diplomatico del tiranno siracusano Ierone.

In un saggio del $2002^{49}$ avevo proposto una lettura diversa di questo avvenimento sulla base di uno studio di entrambe le tradizioni relative agli scoli su Pindaro: ${ }^{50}$ l'attacco di Reggio e di Messana ebbe come conseguenza la sconfitta di Locri, testimoniata dalle dediche votive di Olimpia, ${ }^{51}$ e l'assedio della città. ${ }^{52} \mathrm{Fu}$ solo a questo punto che Ierone inviò come ambasciatore il cognato Cromio, minacciò una guerra contro Reggio e bloccò l'assedio e la conquista reggina della città di Locri: la precedente vittoria reggina venne vanificata così dal tempestivo intervento siracusano.

È il poeta Pindaro che ai versi $18-20$ della seconda Pitica, ${ }^{53}$ scritta in onore di Ierone, tiranno di Siracusa, ricorda il canto di gratitudine delle fanciulle locresi: ${ }^{54}$

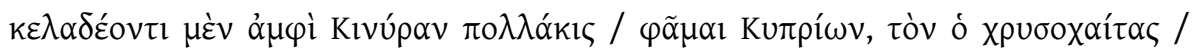

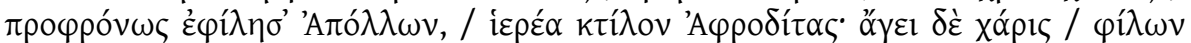

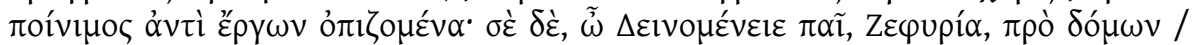

48 Sull'attacco di Reggio contro Locri nel 477 a.C. Cfr. GIOvANNA De SENSI SESTITO, « La Calabria in età arcaica e classica ", in SETTIS (ed.), Storia della Calabria antica, vol. I, p. 227-303, in part. p. 253, 259; EAD., «I Dinomenidi», p. 624, n. 26; MAURIzIo Giangiulio, « Aspetti di storia della Magna Grecia arcaica e classica fino alla guerra del Peloponneso ", in Giovanni Pugliese Carratelli (ed.), Magna Grecia. Lo sviluppo politico, sociale ed economico, Electa, Milano 1987, p. 9-54, in part. p. 41; ID., « Le città di Magna Grecia e Olimpia in età arcaica », in ATTILIo MASTROCinquE (ed.), I grandi santuari della Grecia e l'Occidente, Dipartimento di scienze filologiche e storiche, Trento 1993, p. 93-118, in part. p. 108, n. 45; LURAGHI, "Tirannidi arcaiche», p. 216-224; CoRDIANO, «Espansione territoriale », p. 95-103; D’ANGELo, «L'attacco reggino », p. 9-15; GHEZZI, « I tiranni siracusani », p. 323-328; Giovanna De Sensi Sestito, «Siracusa, le guerre di confine tra Locri e Reggio e il ‘trattato' di Eliano », in Aiônos. Miscellanea di studi storici, 17 (2011-2012), p. 17-48.

49 D'ANGELO, «L'attacco reggino », p. 9-15.

50 Cfr. Scholia Recentia in Pindari Epinicia, ed. ABel Eugen, vol. I: Scholia in Olympia et Pythia, apud S. Calvary et Socios, Budapestini et Berolini 1891; Scholia Vetera in Pindari Carmina, ed. ANDREAs B. DrachmanN, vol. II: Scholia in Phythionicas, Lipsiae 1910 (rist. Verlag Adolph M. Hakkert, Amsterdam 1964).

51 Cfr. SEG, 24, nº 304; 305; 311; 312 .

52 Negli Scholia Recentia al verso 34 della seconda pitica lo scoliasta annota (schol. ad Pind. Pyth. II,

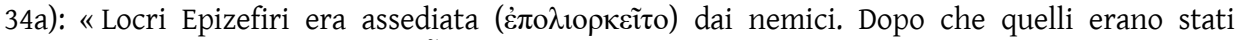

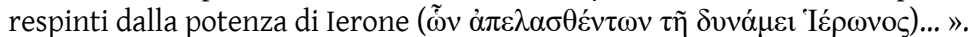

53 Per i problemi relativi alla natura, data e occasione di questo componimento rimando a PINDARO, Le Pitiche, ed. Bruno Gentili, commento a cura di Paola Angeli Bernardini, EtTore Cingano, Bruno Gentili, Pietro Giannini, Fondazione Lorenzo Valla, Arnoldo Mondadori Editore, Milano 1995, intr. p. XLV-LV; p. 43-54.

54 Sulla natura del canto locrese cfr. ANGELA Beldia, Il canto delle vergini locresi. La musica a Locri Epizefirii nelle fonti scritte e nella documentazione archeologica (secoli VI-III a.C.), Serra: Pisa-Roma 2012 (Nuovi saggi, 116), p. 21-22. 


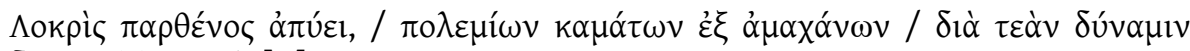
$\delta \rho \alpha \kappa \varepsilon \tilde{c} \zeta \dot{\alpha} \sigma \varphi \alpha \lambda \varepsilon \dot{\varepsilon}[\ldots]$...

Spesso la voce dei Ciprioti celebra Cinira che l'aureochiomato Apollo amò di gran cuore, sacerdote, ministro d'Afrodite; devota gratitudine li guida a compenso d'opere amiche; e te, figlio di Dinomene, celebra innanzi alle case la vergine di Locri Zefiria che dai travagli della guerra ineluttabili ha levato sicuro lo sguardo grazie al tuo potere $[. . .]^{55}$

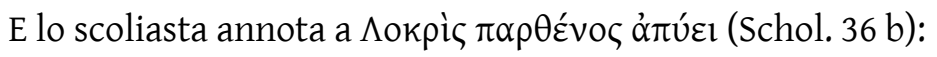

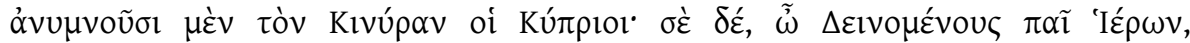

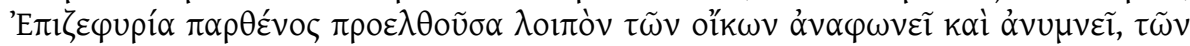

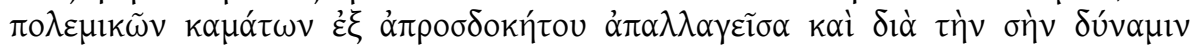

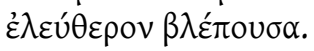

I Ciprioti celebrano Cinira; o Ierone, figlio di Dinomene, le fanciulle locresi, dunque, uscendo fuori dalle loro case ti chiamano e ti invocano, perché liberate inaspettatamente dalle sofferenze dei nemici, grazie alla tua potenza, vedono la libertà.

Nel passo di Pindaro e in quello dello scoliasta non vi sarebbe un'allusione al voto di prostituzione sacra ma solo un riferimento alle sofferenze della guerra: a mio avviso, le fanciulle locresi (che rappresentano probabilmente la comunità civica) vennero salvate dall'assedio di Anassila e di Leofrone, che, una volta concluso, avrebbe comportato sofferenze ben più atroci per Locri Epizefiri che non quelle derivanti da un voto di prostituzione.

Se si guarda la vicenda dalla prospettiva religiosa locrese, la dea Afrodite, a cui i Locresi si erano rivolti ${ }^{56}$ era intervenuta evitando la distruzione di Locri e, pertanto, bisognava da una parte ringraziare il tiranno siracusano, che era divenuto strumento della protezione della dea, ${ }^{57} \mathrm{e}$ dall'altra dare seguito al voto di prostituzione sacra.

55 La traduzione dei versi è tratta da PINDARo, Le Pitiche, p. 63.

56 D'altra parte, già altre volte, i Locresi avevano goduto dell'aiuto di una 'divinità' in un momento di estremo pericolo, ovvero in occasione della battaglia della Sagra quando, secondo la tradizione, i Locresi, in numero nettamente inferiore, erano riusciti a riportare una straordinaria vittoria sui Crotoniati grazie all'intervento dei Dioscuri, figli di Zeus. Sulla battaglia della Sagra cfr. VAN COMPERNOLLE, «Ajax et les Dioscures », p. 733-766; SORDI, «La leggenda dei Dioscuri », p. 47-70; MAURIZIo Giangiulio, «Locri, Sparta, Crotone e le tradizioni leggendarie intorno alla battaglia della Sagra », Mélanges de l'École française de Rome. Antiquité, 95 (1983), p. 473-521; De SenSI SESTITO, «La Calabria in età arcaica», p. 243-246; LuISA Moscati CASTELnUovo, "Sparta e le tradizioni crotoniate e locresi sulla battaglia della Sagra », Quaderni Urbinati di Cultura Classica, 51 (1995), p. 141-163; AlesSANDRo SGOBBI, «Stesicoro, Falaride e la battaglia della Sagra », Acme, 56/3 (2003), p. 3-38.

57 Alla luce di ciò si comprende anche l'accostamento presente nell'ode pindarica tra Cinira e Ierone: le voci $(\varphi \tilde{\alpha} \mu \alpha)$ di Cipro ringraziano Cinira, sacerdote devoto ( $\kappa \tau i ́ \lambda o v)$ ad Afrodite, che 
L'intervento di Ierone, in definitiva, ebbe come conseguenza l'obbligo per i Locresi di prostituire le loro fanciulle in base alla formulazione del voto così come è riportata da Giustino ("Cum Reginorum tyranni Leophronis bello Locrenses premerentur, voverant, si victores forent, ut die festo Veneris virgines suas prostituerent »).

Che il voto fosse stato adempiuto lo rivela, a mio avviso, il sintagma 'Quo voto intermisso', che agli occhi della fonte di Trogo-Giustino o di Dionigi II appare come la causa all'origine dei bella adversa, a cui sono sottoposti i Locresi ad opera dei Lucani: l'espressione 'voto intermisso ${ }^{158}$ non va interpretata come voto trascurato, ma come voto interrotto nel senso che tale pratica rituale da parte delle fanciulle locresi ad un certo momento non venne più continuata. ${ }^{59}$

Anche il voto risalente alla metà del IV secolo a.C. è collegato ad un pericolo militare, ovvero ad una serie di guerre sfavorevoli che i Locresi stavano combattendo contro i Lucani: ciò diventa il pretesto, insieme al voto interrotto del V sec. a.C., per Dionigi II per ripristinare il voto di prostituzione sacra, che nel racconto di Giustino appare come pro forma, una sorta di rito, visto che tutti gli uomini dovevano giurare che nessun uomo avrebbe violato alcuna donna ( omnibus ante iuratis viris, ne quis ullam adtaminet »).

$\mathrm{Fu}$ veramente simbolico il voto di prostituzione sacra pronunciato durante il soggiorno locrese di Dionigi II? Dalla testimonianza di Giustino sembra proprio di sì, anche se le altre fonti letterarie, che ricordano il soggiorno locrese del tiranno, velatamente e implicitamente orientano verso altre direzioni.

Nel racconto di Clearco ${ }^{60}$ ripreso e rielaborato nei contenuti da Eliano ${ }^{61}$, Dionigi II, dopo aver cosparso di rose e di serpilli la casa più grande della città

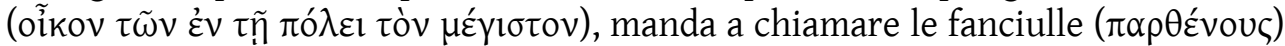

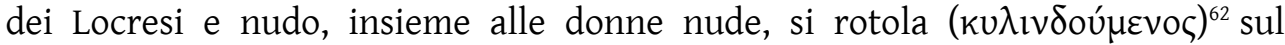
tappeto di fiori.

ha procurato benefici e prosperità all'isola, così come le fanciulle locresi rendono grazie a Ierone, che ha liberato Locri dal pericolo della guerra. Le analogie tra Cinira e Ierone non sono solo di carattere politico ma anche di carattere religioso: Cinira è re dell'isola di Cipro ed è anche sacerdote di Afrodite, Ierone è il signore di Siracusa e anche ierofante delle dee Demetra e Kore. Sul rapporto tra Cinira e Ierone cfr. PInDARo, Le Pitiche, p. 370-373; GHEZzI, «I tiranni siracusani », p. 333-338.

58 Cfr. LoseHAND, « Votum (publicum) intermissum », p. 267-292.

59 Sul significato dell'espressione 'quo voto intermitto', cfr. BRUNo CURRIE, Pindar and the Cult of Heroes, Oxford University Press, Oxford 2005, p. 265-266.

60 Clear. apud Athen. XII 541 C-E fr. 47 Wehrli.

61 Ael. Var. Hist. IX, 8.

62 L'azione del kylindesthai andrebbe intesa come volteggiare o danzare. Cfr. BeLLiA, Il canto delle vergini locresi, p. 22, n. 15. 
Strabone ${ }^{63}$ descrive un curioso quadro in cui il tiranno siracusano riunisce le fanciulle $(\pi \alpha \rho \theta \varepsilon ́ v o u \varsigma)$ più belle di Locri nei banchetti e le obbliga a inseguire nude, con sandali di diversa altezza, alcune colombe lasciate libere nella sala.

Questi giochi erotici potrebbero apparire agli occhi del lettore moderno come le fantasie di una mente perversa o come semplici particolari aneddotici delle fonti, interessate a mettere in evidenza la mollezza del tiranno ( fosse per i tratti fortemente religiosi degli elementi che caratterizzano queste scene: rose, serpilli e colombe rimandano, infatti, al culto di Afrodite. ${ }^{64}$

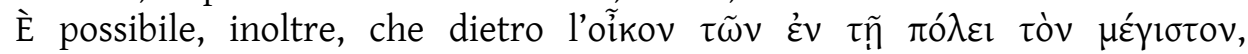
menzionato da Clearco, possa celarsi un riferimento al tempio di Venere, di cui parla Giustino.

Non c'è ombra di dubbio che i racconti di Giustino e di Clearco-Eliano appartengano a tradizioni letterarie autonome e fortemente tendenziose ${ }^{65}$ che evidentemente erano interessate a mettere in luce aspetti differenti del soggiorno locrese del tiranno siracusano: ${ }^{66}$ nello specifico mentre la fonte di Trogo-Giustino (probabilmente Teopompo) ${ }^{67}$ presenta il voto come un atto simbolico con cui il tiranno intende appropriarsi delle ricchezze dei Locresi, i racconti di Clearco-Eliano e di Strabone presentano scene aneddotiche a forti tinte religiose, in cui le parthenoi locresi vengono comunque violate. ${ }^{68}$

Infine un aspetto che accomuna i due voti secondo la tradizione trogiana è che entrambi siano messi in relazione con la dea Afrodite/Venere: ${ }^{69}$ il voto del 477 a.C. è inserito in un die festo Veneris, quello dell'epoca dionigiana presenta due cornici, quella del lupanar e quella di un templum Veneris.

63 Strab. VI 18 C 259.

64 Cfr. ToRelli, « Considerazioni sugli aspetti », p. 96-97; GHEZZI, « I tiranni siracusani », p. 343-344.

65 D’ANGELo, « La tradizione letteraria », p. 188-191, n. 40-41, 43-44.

66 Ibid., p. 188; GHEZzI, «I tiranni siracusani », p. 345.

67 Teopompo di Chio, vissuto intorno alla metà del IV secolo a.C., è la fonte di Trogo-Giustino per quanto riguarda il soggiorno locrese di Dionigi II: i frammenti, che ci sono rimasti di Teopompo su Dionigi II e sui suoi familiari, si avvicinano per contenuto ai racconti di Giustino e mostrano Dionigi e i suoi come degli ubriaconi e dei dissoluti, violatori di donne e di bambini. Il frammento di Teopompo (FgrHist 115 FF 283a-b) sulla malattia agli occhi, che aveva colpito Dionigi II a causa della sua smodatezza nel bere, trova un chiaro riscontro proprio in un altro passo di Giustino (XXI 2,1). Cfr. MARTA SORDI, in Diodori Siculi Bibliothecae liber XVI, ed. MARTA SORDI, La Nuova Italia, Firenze 1969 (Biblioteca di studi superiori, 56), p. 39 dell'introduzione; PAUL PÉDECH, Trois historiens méconnus. Théopompe-Duris-Phylarque, Les belles lettres, Paris 1989 (Collection d'études anciennes, 119), p. 164-169, n. 10; Giovanna De Sensi SestiTo, « I due Dionisii e la nascita della confederazione brettia », in Giovanna De Sensi SeStito (ed.), I Brettii, t. I: Cultura, lingua, e documentazione storico-archeologica. Atti del I corso seminariale - Rossano, 20-26 Febbraio, Rubbettino, Soveria Mannelli 1995, p. 33-71, in part. p. 46, n. 62; Muccioli, Dionigi II, p. 89; D’ANGELO, « La tradizione letteraria », p. 182.

68 Cfr. GHEZZI, « I tiranni siracusani », p. 346.

69 Sul rapporto molto stretto tra il culto di Afrodite nel mondo greco e la pratica della prostituzione sacra cfr. TORELLI, « I culti di Locri », p. 152-154. 
La tradizione letteraria relativa alla presenza di un culto ad Afrodite a Locri Epizefiri è stata confermata dalle esplorazioni archeologiche effettuate sia in località Marasà, dove sono stati trovati i resti di un tempio ionico, sia nell'area di Centocamere, ${ }^{70}$ dove gli scavi hanno evidenziato una serie di oikoi, che costituiscono la cosiddetta Stoà a U e, a breve distanza da questa, un sacello, ${ }^{71}$ che rappresentava il luogo di culto vero e proprio della divinità. ${ }^{72}$

La Stoà a U si sviluppava su tre lati ed era costituita da un cortile centrale, occupato da piccoli bothroi, da una sala sul fondo e da ambienti rettangolari ai lati ${ }^{73}$ : proprio le stanze laterali presentano ambienti, che ben si adatterebbero alla pratica della prostituzione sacra (cfr. Fig. 1). ${ }^{74}$

Inoltre la presenza del culto di Afrodite a Locri è testimoniata anche da alcune iscrizioni, ritrovate a Locri, ${ }^{75}$ che riportano il nome della dea: ${ }^{76}$ la maggior parte di queste iscrizioni sono state reperite proprio nell'area di Centocamere-Marasa che probabilmente doveva essere il luogo principale del culto rivolto alla dea (cfr. Fig. 2). ${ }^{77}$

70 La presenza di un culto ad Afrodite è attestata anche presso il santuario della Mannella dove era venerata la dea Persefone cfr. GuarduccI, « Due pezzi insigni », p. 4; Bellia, Il canto delle vergini locresi, p. 58.

71 La Bagnasco identifica in questo sacello il templum Veneris, di cui parla Giustino (XXI 3) o il voó che compare nel già ricordato epigramma di Nosside (Anth. Pal. IX, 332). Cfr. MARCELla BARRA BAGNASCO, "Nuovi documenti sul culto di Afrodite a Locri Epizefiri », Parola del Passato, 250 (1990), p. 42-53, in part. p.51; MARIO TORELLI, Dei e artigiani. Archeologie delle colonie greche d'Occidente, GLF editori Laterza, Roma-Bari 2011, p. 77-88.

72 Cfr. TORELLI, «I culti di Locri », p. 147-156; BARRA BAGNASCO, « Nuovi documenti sul culto », p. 4253.

73 Cfr. ToRelli, « I culti », p. 597-607; BelLiA, Il canto delle vergini locresi, p. 65-68.

74 Per il Torelli la Stoà ad U potrebbe identificarsi con il lupanar di Giustino (XXI 3). Cfr. TorelLI, « I culti di Locri», p. 154-155; ID., "Considerazioni sugli aspetti», p. 98. Gli studiosi hanno interpretato gli oikoi in maniera diversa: sale di banchetto (Graf), celle adibite alla prostituzione sacra (Guarducci), ambienti dedicati a forme di culto connesse con pranzi iniziatici (Barra Bagnasco). Cfr. GRAF, «Culti e credenze », p. 178-179; GUARDUCCI, « Due pezzi insigni », p. 10. Cfr. BARRA BAGNASCO, « Nuovi documenti sul culto », p. 48, n. 14.

75 Sempre a Locri è stata ritrovata una iscrizione presso Centocamere con dedica a Kybala, la Cibele microasiatica, che la tradizione ionica antica identificava con Afrodite. Cfr. MARGHERITA GuARDUCCI, «Cibele in un'epigrafe arcaica di Locri Epizefiri », Klio, 52 (1970), p. 133-138; ToRelLI, " I culti di Locri », p. 150; AMANTINI, " Ancora sulla prostituzione sacra », p. 44; ToReLl, « I culti », p. 599; Felice Costabile, «Defixiones da Locri Epizefiri: nuovi dati sui culti, sulla storia e sulle istituzioni », Minima Epigraphica et Papyrologica, 2 (1999), p. 22-76, in part. p. 25-29.

76 BarRa Bagnasco, "Nuovi documenti sul culto», p. 42-53; Marcella BarRa Bagnasco, "Nuova dedica locrese ad Afrodite ", Parola del Passato, 250 (1990), p. 62-63; LAvinio Del Monaco, Iscrizioni greche d'Italia, Quasar, Roma 2013, p. 110-122; Enzo LipPoLIs, « Alcune osservazioni sull'uso e sulla diffusione della coroplastica rituale nei depositi dell'Italia meridionale: il caso di Locri Epizefiri », Polymnia. Studi di archeologia, 6 (2014), p. 55-93, in part. p. 74, n. 61.

77 Lippolis, «Alcune osservazioni sull'uso », p. 75. 
Questi dati archeologici risultano molto interessanti se messi a confronto con la tradizione letteraria, con la quale presentano diversi punti di contatto: il tempio e il lupanare, ricordati da Giustino (XXI 3), potrebbero trovare una corrispondenza archeologica rispettivamente nel sacello e nella Stoà ad U dove erano presenti gli oikoi (le camerette), così come il die festo Veneris, menzionato dallo stesso Giustino, troverebbe una conferma nel culto di Afrodite, testimoniato da diverse iscrizioni religiose.

Inoltre la Stoà a $\mathrm{U}$, posta fuori dalle mura della città e vicino al mare, poteva prestarsi bene all'attività delle sacre prostitute, che venivano lasciate libere ai

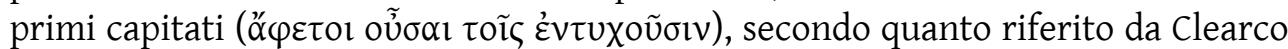
di Soli nel frammento di Ateneo (XII 516 B). ${ }^{78}$

Dall'esame delle fonti letterarie e dalle rilevanze archeologiche possiamo trarre, a mio avviso, le seguenti conclusioni:

1. L'analisi delle fonti letterarie e archeologiche ci consente di affermare con certezza che a Locri Epizefiri esisteva la prostituzione sacra, legata al culto di Afrodite: la concisa testimonianza di Clearco, relativa alla presenza della prostituzione sacra in Lidia, a Locri e a Cipro, ${ }^{79}$ può essere considerata attendibile ${ }^{80}$ non solo perché Clearco, vivendo intorno alla metà del IV sec. a.C., poteva disporre di fonti di prima mano relative alla storia di Locri Epizefiri ma anche perché il resoconto di Clearco sul soggiorno locrese di Dionigi II, almeno nelle linee generali, sembra confermato dai resoconti di Strabone e di Trogo-Giustino. L'epigramma della poetessa Nosside sulla statua in oro di Afrodite, donata dall'etera Poliarchide, conferma, a mio avviso, che la pratica della prostituzione sacra fosse ancora in atto tra la fine del IV e l'inizio del III sec. a.C.

2. La tradizione letteraria sulle violenze sessuali di Dionigi II e sulla fine atroce che i Locresi destinarono alla famiglia del tiranno ha sicuramente preso spunto dai rituali collegati con la prostituzione sacra oltre che dai racconti in qualche modo ad essa connessi (Lycofr. Alex. 1155-1159; Tzetze Chil. 5 728-745).

78 TORELLI, « I culti », p. 602-603.

79 Secondo alcuni studiosi il testo di Clearco descrive il fenomeno della prostituzione come una pratica abituale, che probabilmente interessava gli elementi servili e gli strati bassi della popolazione, mentre i due voti del 477 e del 351-346, tramandati da Giustino, coinvolsero certamente anche la classe aristocratica. Cfr. Musti, «Problemi della storia », p. 67; AMANTINI, «Ancora sulla prostituzione sacra », p. 50; CAPPELLETTI, « Esclusive notizie », p. 488.

80 Il valore documentario di Clearco di Soli è testimoniato dallo stesso Ateneo che, alla fine dei Deipnosofisti (XV $701 \mathrm{C}$ ), lo definisce oủdzvò $\mu \alpha \theta \eta \tau \tilde{\omega} v$. Cfr. Giuseppe ZeCChinI, La cultura storica di Ateneo, Vita e pensiero, Milano 1989, p. 198208. 
3. I due voti di prostituzione sacra, a cui fa riferimento in maniera esplicita il racconto di Trogo-Giustino e che coinvolsero le donne aristocratiche, ebbero un esito simile: quello del 477 a.C. non solo fu pronunciato, ma venne anche attuato e, dopo alcuni anni, evidentemente interrotto (così, a mio avviso, va interpretata l'espressione 'quo voto intermisso'). Per quanto riguarda il voto risalente all'epoca del soggiorno locrese di Dionigi II, di cui ci sono giunti sia il racconto dettagliato, ma non sempre lineare di Giustino, sia gli echi presenti nelle altre fonti, la scena del tappeto cosparso di fiori e di serpilli, su cui si rotolano Dionigi II e le fanciulle locresi nella casa più grande della città, ${ }^{81}$ lo ius primae noctis che il tiranno esercita a Locri, lo strano gioco in cui le fanciulle locresi sono costrette dal tiranno ad inseguire delle colombe, ${ }^{82}$ l'uso della forma

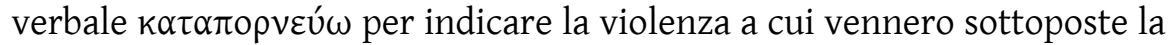
moglie e le figlie del tiranno, prima di essere uccise, ${ }^{83}$ sembrano orientare per una attuazione del voto della metà del IV secolo a.C., anche se i contorni e le circostanze, in cui tale voto venne adempiuto, non sono del tutto chiari.

81 Clear. apud Athen. XII 541 C-E fr. 47 Wehrli; Ael. Var. Hist. IX, 8.

82 Strab. VI 18 C 259.

83 Ael. Var. Hist. IX, 8; Strab. VI 18 C 259; Plut. Timol. 13. 


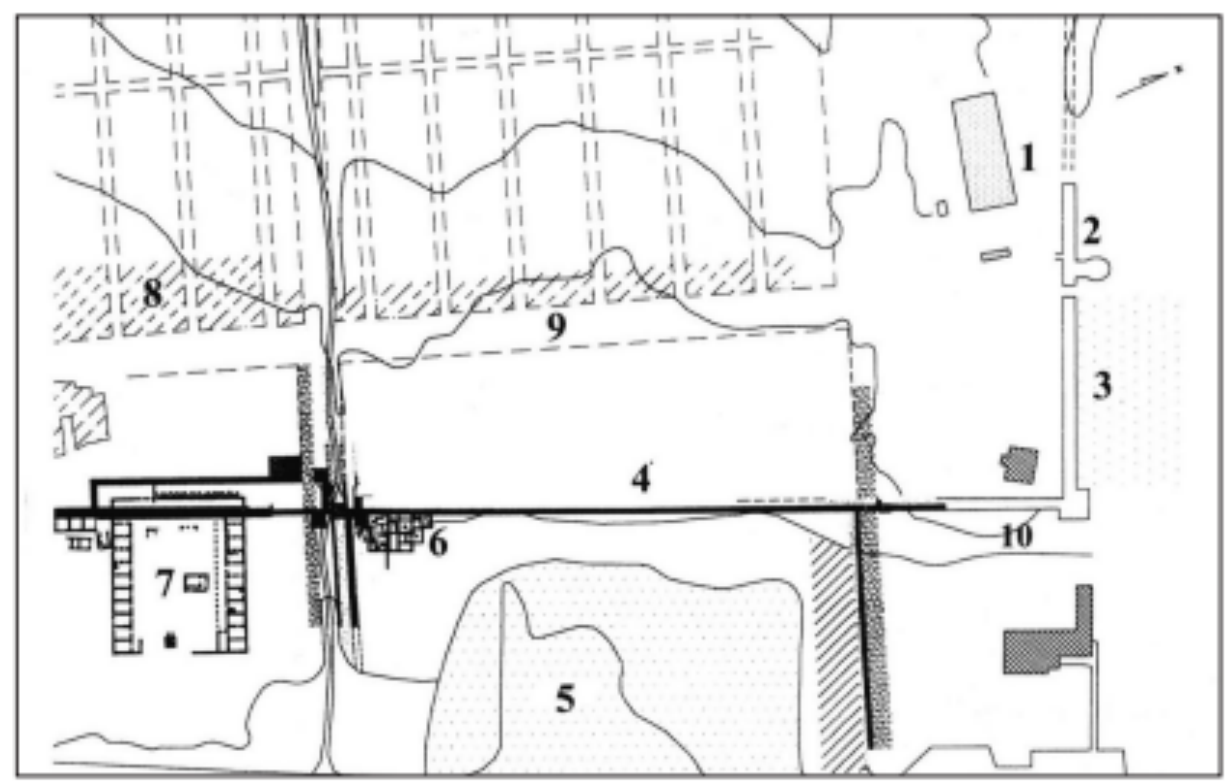

Fig. 1. Planimetria dell'area di Centocamere-Marasà. 1: Tempio ionico; 2: Mura e torre; 3: Thesmophorion; 4: Mura; 5: Area portuale; 6: Sacello di Afrodite e Casa dei leoni - Adonion; 7: 'Stod $a U$ '; 8: Isolati di abitazione di Centocamere; 9: Plateia C; 10: Stipe di Zeus fulminante. (In MARIO TORELli, Dei e artigiani. Archeologie delle colonie greche d'Occidente, GLF editori Laterza, Roma-Bari 2011, p. 79, fig. 46). 

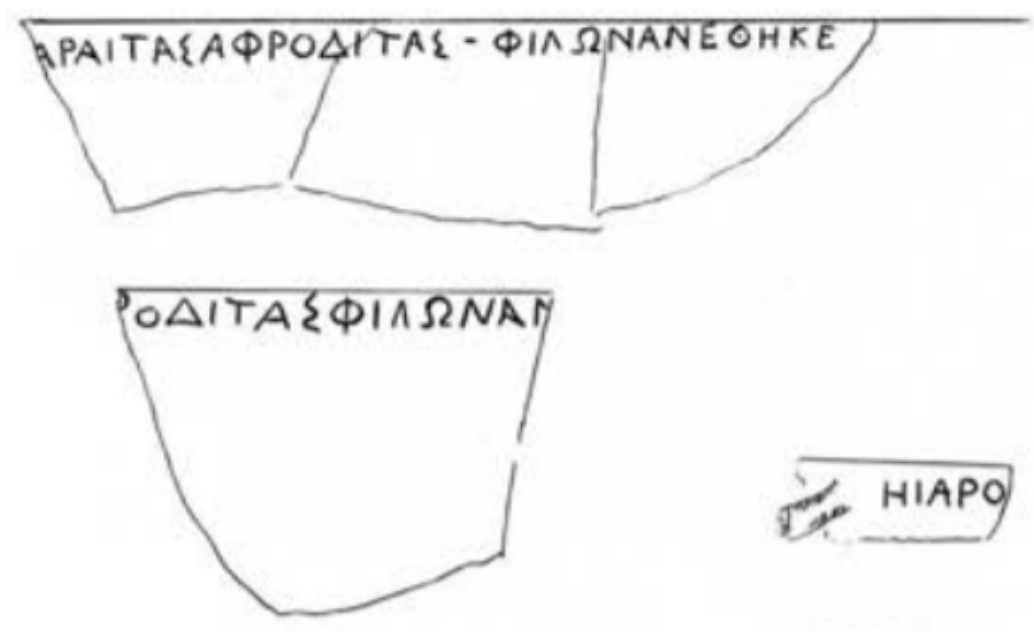

HIAPQ
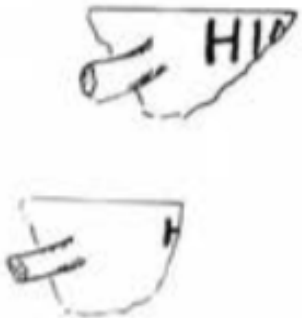

Fig. 2: Frammenti di skyphoi a vernice nera con iscrizioni dal bothros n. 24 di Locri, Centocamere. (In MARGHERITA GUARDUCCI, «Due pezzi insigni del museo nazionale romano: il 'Trono Ludovisi' e l'AAcrolito Ludovisi' », Bollettino d'arte, 33-34 (1985), p. 4.). 